\title{
Some bounds of the generalized $\mu$-scrambling indices of primitive digraphs with $d$ loops
}

\author{
Ling Zhang ${ }^{1}$, Gu-Fang Mour ${ }^{2 *}$ Feng Liu ${ }^{3}$ and Zhong-Shan Li ${ }^{4}$
}

\section{"Correspondence:}

mougufang1010@163.com

${ }^{2}$ College of Applied Mathematics, Chengdu University of Information

Technology, Sichuan, China

Full list of author information is

available at the end of the article

\section{Springer}

\begin{abstract}
In 2010, Huang and Liu introduced a useful parameter called the generalized $\mu$-scrambling indices of a primitive digraph. In this paper, we give some bounds for $\mu$-scrambling indices of some primitive digraphs with $d$ loops and the digraphs attained the sharp upper bounds are provided.
\end{abstract}

MSC: $05 C 20 ; 05 C 50$

Keywords: Generalized $\mu$-scrambling index; Primitive digraph; Bound; Scrambling index

\section{Introduction}

For the research on the competition index, $m$-competition index, the scrambling index and the generalized $\mu$-scrambling index, please refer to $[1-3,5,6,8,9]$ and $[7,11]$, respectively. Cho et al. [6] defined the $m$-step competition graph of a digraph which is an extension of a competition graph. In 2009, Akelbek and Kirkland [2] defined and studied the scrambling index of a primitive digraph and provided an upper bound on the scrambling index of a primitive digraph. The $m$-competition index of a primitive digraph was introduced by Kim [8]. Kim investigated the $m$-competition index of a primitive digraph and gave an upper bound for the $m$-competition indices of primitive digraphs. In 2010, Huang and Liu [7] gave the definition of the generalized $\mu$-scrambling indices for a primitive digraph which are a generalization of the scrambling index and $m$-competition index and they provided some bounds for the generalized $\mu$-scrambling indices of some primitive digraphs. In this paper, we give some bounds for $\mu$-scrambling indices of some primitive digraphs.

The outline of this paper is as follows: Some notation and notions used throughout this paper are introduced in Sect. 2. In Sect. 3, we study the generalized $\mu$-scrambling indices of the primitive digraphs with $d$ loops.

\section{Definitions and terminology}

In this section, we introduce some definitions, notations which are needed to use in the presentations and proofs of our main results in this paper.

(c) The Author(s) 2021. This article is licensed under a Creative Commons Attribution 4.0 International License, which permits use, sharing, adaptation, distribution and reproduction in any medium or format, as long as you give appropriate credit to the original author(s) and the source, provide a link to the Creative Commons licence, and indicate if changes were made. The images or other third party material in this article are included in the article's Creative Commons licence, unless indicated otherwise in a credit line to the material. If material is not included in the article's Creative Commons licence and your intended use is not permitted by statutory regulation or exceeds the permitted use, you will need to obtain permission directly from the copyright holder. To view a copy of this licence, visit http://creativecommons.org/licenses/by/4.0/. 
A digraph $D$ consists of a nonempty set $V=V(D)$ and an arc set $E=E(D)$. In $D$, loops are permitted but multiple arcs are not. A path $P=x \rightarrow y$ is a sequence of edges $\left\{\left(x, v_{1}\right),\left(v_{1}, v_{2}\right), \ldots,\left(v_{k-1}, y\right)\right\}$ in which all vertices are distinct. A cycle $C$ is a closed path with the first and the last vertices coincided. A walk from $x$ to $y$ is a sequence of arcs: $e_{1}, e_{2}, \ldots, e_{k}$ such that the terminal vertex of $e_{i}$ is the same as the initial vertex of $e_{i+1}$ for $i=1,2, \ldots, k-1$, denoted by $W=x \rightarrow y$. The length of a walk or cycle is the number of arcs. A walk $W=x \rightarrow y$ of length $k$ is denoted by $x \stackrel{k}{\rightarrow} y$. A cycle of length $l$ is denoted by $C_{l}$. The girth of $D$ which has at least one cycle, is the length of a shortest cycle in $D$.

A digraph $D$ is primitive with a walk of length $k$ from each vertex $x$ to each vertex $y$ (not necessarily distinct). The digraph $D$ is primitive if and only if $D$ is strongly connected and the greatest common divisor of the lengths of its cycles is 1 (see [4]). For a positive integer $s$, the sth power of $D$, denoted by $D^{(s)}$, is the digraph on the same vertex set $V(D)$ and with an $\operatorname{arc}$ from $i$ to $j$ if and only if $i \stackrel{s}{\rightarrow} j$ in $D$. The scrambling index $k(D)$ of a primitive digraph $D$ is the smallest positive integer $k$ such that, for every pair of vertices $u$ and $v$, there exists a vertex $w$ such that $u \stackrel{k}{\rightarrow} w$ and $v \stackrel{k}{\rightarrow} w$ in $D$ (see [2]).

Let $D$ be a digraph with vertex set $V$ and let $k$ be a positive integer. A vertex $w$ of $D$ is a $k$-step common prey for $u$ and $v$ if $u \stackrel{k}{\rightarrow} w$ and $v \stackrel{k}{\rightarrow} w$. The $k$-step $m$-competition graph of $D$ has the same vertex set of $D$ and an edge between vertices $u$ and $v$ if and only if there are at least $m$ distinct vertices $v_{1}, \ldots, v_{m}$ in $D$ such that $u \stackrel{k}{\rightarrow} v_{i}$ and $v \stackrel{k}{\rightarrow} v_{i}$ for $i=1,2, \ldots, m$ (see [6]). The m-competition index $c(D, m)$ of a primitive digraph $D$ is the smallest positive integer $k$ such that, for every pair of vertices $u$ and $v$, there are $m$ distinct vertices $v_{1}, \ldots, v_{m}$ in $D$ such that $u \stackrel{k}{\rightarrow} v_{i}$ and $v \stackrel{k}{\rightarrow} v_{i}$ for $i=1,2, \ldots, m$ (see [2]). That is to say, the $m$-competition index of $D$ is the smallest positive integer $k$ such that the $k$-step $m$-competition graph is complete.

Let $P_{n}$ denote the set of all primitive digraphs of order $n$.

Definition 2.1 ([7]) Let $D \in P_{n}$, and $\lambda, \mu$ be integers with $1 \leq \lambda, \mu \leq n$. For $X \subseteq V(D)$, let $k_{X}^{(\mu)}$ be the smallest positive integer $m$ such that there exist $\mu$ vertices $w_{1}, w_{2}, \ldots, w_{\mu}$ of $D$ such that $x \stackrel{m}{\rightarrow} w_{i}(i=1,2, \ldots, \mu)$ in $D$ for every vertex $x$ of $X$. Then

$$
\begin{aligned}
& h(D, \lambda, \mu):=\min \left\{k_{X}^{(\mu)} \mid X \subseteq V(D) \text { and }|X|=\lambda\right\} \text { and } \\
& k(D, \lambda, \mu):=\max \left\{k_{X}^{(\mu)} \mid X \subseteq V(D) \text { and }|X|=\lambda\right\}
\end{aligned}
$$

are called the $\lambda$ th lower and upper $\mu$-scrambling indices of $D$, respectively. For convenience, let $k_{X}(D):=k_{X}^{(1)}(D), h(D, \lambda):=h(D, \lambda, 1)$ and $k(D, \lambda):=k(D, \lambda, 1)$.

Since $k(D, 2)=k(D)$, in [7] Huang and Liu called $h(D, \lambda, \mu)$ and $k(D, \lambda, \mu)$ the generalized $\mu$-scrambling indices, $h(D, \lambda)$ and $k(D, \lambda)$ the generalized scrambling indices of $D$ in $P_{n}$. As $k(D, 2, m)=c(D, m)$, the generalized $\mu$-scrambling indices are also generalizations of the $m$-competition index.

\section{Generalized $\boldsymbol{\mu}$-scrambling indices}

In [7], Huang and Liu investigated generalized scrambling indices of the primitive digraphs with $d$ loops. In this section, we study the generalized $\mu$-scrambling indices of the primitive digraphs with $d$ loops. 
For a vertex subset $X \subseteq V(D)$, define $R_{t}^{D}(X)$ to be the set of vertices in $D$ reachable from some vertices in $X$ via a walk of length $t$.

Let $d$ be an integer with $1 \leq d \leq n$ and let $P_{n}(d)$ be the class of primitive digraphs with $n$ vertices and $d$ loops. Let $L_{n, d}(1 \leq d \leq n)$ be the digraph with vertex set $V\left(L_{n, d}\right)=$ $\{1,2, \ldots, n\}$ and arc set

$$
E\left(L_{n, d}\right)=\{(i, i+1) \mid 1 \leq i \leq n-1\} \cup\{(n, 1)\} \cup\{(i, i) \mid n-d+1 \leq i \leq n\} .
$$

Theorem 3.1 Let $D \in P_{n}(d)$ and $1 \leq \lambda, \mu \leq n$.

$$
h(D, \lambda, \mu) \leq \begin{cases}\lambda+\mu-2, & \lambda+\mu<n+1, \\ n-1, & d \geq \lambda, \lambda+\mu \geq n+1, \\ n-1, & d<\lambda, n+1 \leq \lambda+\mu \leq n+d, \\ \lambda+\mu-d-1, & d<\lambda, \lambda+\mu>n+d,\end{cases}
$$

and the bound can be attained by the digraph $L_{n, d}$.

Proof Since $D \in P_{n}(d)$, there exists a loop vertex $u$ such that there is a set $Y$ of $\lambda-1$ vertices whose distances to $u$ are at most $\lambda-1$. If $\lambda+\mu<n+1$, let $X=Y \cup\{u\}$. Then $|X|=\lambda$. Since $D$ is strongly connected and $u$ is a loop vertex, the minimum number of vertices that can be reached from $u$ at $(\mu-1)$-step in $D$ is $\mu$. Therefore, $\left|\bigcap_{x \in X} R_{\lambda+\mu-2}^{D}(\{x\})\right| \geq \mu$, which implies that $h(D, \lambda, \mu) \leq \lambda+\mu-2$.

If $d \geq \lambda$ and $\lambda+\mu \geq n+1$, let $X$ be a vertex set which contains $\lambda$ loop vertices. Since each vertex in $X$ is a loop vertex, we have $R_{n-1}^{D}(X)=V(D)$. Therefore, $\left|R_{n-1}^{D}(X)\right|=|V(D)|=$ $n \geq \mu$, which implies that $h(D, \lambda, \mu) \leq \lambda+\mu-2$.

If $d<\lambda$, let $Z$ be the vertex set of $d$ loop vertices and $X_{i} \subseteq(V(D) \backslash Z)$ be the vertex set of $x_{i}$ vertices whose shortest distance to vertices of $Z$ is $i$, where $1 \leq i \leq \lambda-d$. Assume $\sum_{i=1}^{r} x_{i} \leq \lambda-d<\sum_{i=1}^{r+1} x_{i}$, where $1 \leq r \leq \lambda-d$. Let $X=Z \cup X_{1} \cup \cdots \cup X_{r} \cup \bar{X}_{r+1}$, where $\bar{X}_{r+1} \subseteq X_{r+1}$ contains $\bar{x}_{r+1}$ vertices and $\sum_{i=1}^{r} x_{i}+\bar{x}_{r+1}=\lambda-d$. Then $|X|=\lambda$.

If $d<\lambda$ and $n+1 \leq \lambda+\mu \leq n+d$, since $R_{n-1}^{D}(Z)=V(D)$ and $R_{n-1}^{D}\left(X_{1} \cap \cdots \cap X_{r} \cap \bar{X}_{r+1}\right)$ contains at least $n-\sum_{i=1}^{r} x_{i}-\bar{x}_{r+1}=n-\lambda+d$ vertices, we have $\left|R_{n-1}^{D}(X)\right| \geq n-\lambda+d \geq \mu$. Therefore, $h(D, \lambda, \mu) \leq \lambda+\mu-2$.

If $d<\lambda$ and $\lambda+\mu>n+d$, let $k=\lambda+\mu-n-d$. Notice that $R_{\lambda+\mu-d-1}^{D}\left(X_{i}\right)=V(D)$, for $1 \leq i \leq k$. If $k \geq r+1$, then $\left|R_{\lambda+\mu-d-1}^{D}(X)\right|=|V(D)|=n \geq \mu$.

If $k<r+1$, then $R_{\lambda+\mu-d-1}^{D}(X)=\bigcap_{i=k+1}^{r}\left[R_{\lambda+\mu-d-1}^{D}\left(X_{i}\right)\right] \cap R_{\lambda+\mu-d-1}^{D}\left(\bar{X}_{r+1}\right)$. Since $\bigcap_{i=k+1}^{r}\left[R_{\lambda+\mu-d-1}^{D}\left(X_{i}\right)\right] \cap R_{\lambda+\mu-d-1}^{D}\left(\bar{X}_{r+1}\right)$ contains at least $n-\left(\sum_{i=k+1}^{r} x_{i}+\bar{x}_{r+1}\right)$ vertices, we have $\left|R_{\lambda+\mu-d-1}^{D}(X)\right| \geq n-\left(\lambda-d-\sum_{i=1}^{k} x_{i}\right) \geq n-\lambda+d+k=n-\lambda+d+(\lambda+\mu-n-d)=\mu$. We thus arrive at $h(D, \lambda, \mu) \leq \lambda+\mu-2$.

On the other hand, consider the digraph $L_{n, d}$. Lex $X$ be a vertex set with $\lambda$ vertices. If $\lambda+$ $\mu<n+1$, since $R_{\lambda+\mu-3}^{L_{n, d}}(i)=\{i, \ldots, n, \ldots, \lambda+\mu+i-n-3\}$, for $n-d+1 \leq i \leq n$ and $R_{\lambda+\mu-3}^{L_{n, d}}(i)=$ $\{n-d+1, \ldots, n, \ldots, \lambda+\mu+i-n-3\}$, for $1 \leq i \leq n-d+1$, we obtain $\left|\bigcap_{x \in X} R_{\lambda+\mu-3}^{L_{n, d}}(\{x\})\right| \leq$ $\mu-1$.

Noticing that $R_{n-2}^{L_{n, d}}(i)=\{i, \ldots, n, \ldots, i-2\}$, for $n-d+1 \leq i \leq n$, and $R_{n-2}^{L_{n, d}}(i)=\{n-d+$ $1, \ldots, n, \ldots, i-2\}$, for $1 \leq i<n-d+1$. If $d \geq \lambda$ and $\lambda+\mu \geq n+1$, then, for any vertex $x \in X, R_{n-2}^{L_{n, d}}(\{x\})$ contains at most $n-2$ vertices. Therefore, $\bigcap_{x \in X} R_{n-2}^{L_{n, d}}(\{x\})$ contains at 
most $n-\lambda-1$ vertices. As $\lambda+\mu \geq n+1$, we have $\left|\bigcap_{x \in X} R_{n-2}^{L_{n, d}}(\{x\})\right| \leq \mu-2$. Consequently, we obtain $h(D, \lambda, \mu)=\lambda+\mu-2$.

If $d<\lambda$ and $n+1 \leq \lambda+\mu \leq n+d$, we have for any set $X$, there is at least one vertex $x \in X$ such that $R_{n-2}^{L_{n, d}}(\{x\})$ contains at most $n-3$ vertices. Thus, $\bigcap_{x \in X} R_{n-2}^{L_{n, d}}(\{x\})$ contains at most $n-\lambda-2$ vertices. Since $\lambda+\mu \geq n+1$, we obtain $\left|\bigcap_{x \in X} R_{n-2}^{L_{n, d}}(\{x\})\right| \leq \mu-3$. Therefore, $h(D, \lambda, \mu)=\lambda+\mu-2$.

If $d<\lambda$ and $\lambda+\mu>n+d$, we have $R_{\lambda+\mu-d-2}^{L_{n, d}}(i)=V\left(L_{n, d}\right)$, for $2 n+2-\lambda-\mu \leq i \leq n$, and $R_{\lambda+\mu-d-2}^{L_{n, d}}(i)=\{n-d+1, \ldots, n, \ldots, \lambda+\mu+i-n-d-2\}$, for $1 \leq i<2 n+2-\lambda-\mu$. Thus, for any vertex set $X$ of $\lambda$ vertices, there is a set $Y \subseteq X$ of at least $n+1-\mu$ vertices, such that, for any vertex $y \in Y, R_{\lambda+\mu-d-2}^{L_{n, d}}(\{y\})$ contains at most $\lambda+\mu+y-n-2$ vertices, where $1 \leq y<2 n+2-\lambda-\mu$. It follows that $\bigcap_{y \in Y} R_{\lambda+\mu-d-2}^{L_{n, d}}(\{y\})$ containing at most $\mu-1$ vertices. Therefore, $\left|\bigcap_{x \in X} R_{\lambda+\mu-d-2}^{L_{n, d}}(\{x\})\right| \leq\left|\bigcap_{y \in Y} R_{\lambda+\mu-d-2}^{L_{n, d}}(\{y\})\right| \leq \mu-1$. It follows that $h(D, \lambda, \mu)=\lambda+\mu-2$. This completes the proof.

Lemma 3.2 ([10]) Let $D \in P_{n}(d)$ and $\emptyset \neq X \subseteq V(D)$. Then, for nonnegative integers $i, j, t$, $k$, we have $R_{i}^{D}(X)=R_{i-j}^{D}\left(R_{j}^{D}(X)\right)$ for $i \geq j$, and $\left|\bigcup_{t=0}^{k} R_{t}^{D}(X)\right| \geq \min \{|X|+k, n\}$.

Theorem 3.3 Let $D \in P_{n}(d)$ and $1 \leq \lambda, \mu \leq n$. Then

$$
k(D, \lambda, \mu) \leq \begin{cases}n-\left\lceil\frac{d-\mu+1}{\lambda}\right\rceil, & \mu \leq d, \\ n+\mu-d-1, & \mu>d\end{cases}
$$

and the bound can be attained by the digraph $L_{n, d}$.

Proof Let $X \subseteq V(D)$ be a vertex set of any $\lambda$ vertices. Set $X=\left\{v_{1}, v_{2}, \ldots, v_{\lambda}\right\}$.

Case 1. If $\mu \leq d$.

For any vertex $v_{i} \in X$, since $D \in P_{n}(d) \subseteq P_{n}$, by Lemma 3.2,

$$
\left|\bigcup_{t=0}^{n-\left\lceil\frac{d-\mu+1}{\lambda}\right\rceil} R_{t}^{D}\left(\left\{v_{i}\right\}\right)\right| \geq n-\left\lceil\frac{d-\mu+1}{\lambda}\right\rceil+1,
$$

where $i=1,2, \ldots, \lambda$. Let $\frac{d-\mu+1}{\lambda}=k^{\prime}+a$ where $k^{\prime}$ is a nonnegative integer and $0 \leq a<1$. Therefore, if $0<a<1$,

$$
\left|\bigcap_{i=1}^{\lambda}\left[\bigcup_{t=0}^{n-k^{\prime}-1} R_{t}^{D}\left(\left\{v_{i}\right\}\right)\right]\right| \geq \lambda\left(n-k^{\prime}\right)-n(\lambda-1) \geq n-d+\mu+\lambda a-1 .
$$

Since $\lambda a \geq 1$,

$$
\left|\bigcap_{i=1}^{\lambda}\left[\bigcup_{t=0}^{n-k^{\prime}-1} R_{t}^{D}\left(\left\{v_{i}\right\}\right)\right]\right| \geq n-d+\mu .
$$

If $a=0$,

$$
\left|\bigcap_{i=1}^{\lambda}\left[\bigcup_{t=0}^{n-k^{\prime}-1} R_{t}^{D}\left(\left\{v_{i}\right\}\right)\right]\right| \geq \lambda\left(n-k^{\prime}+1\right)-n(\lambda-1) \geq n-d+\mu+\lambda-1 .
$$


Since $\lambda \geq 1$, we have

$$
\left|\bigcap_{i=1}^{\lambda}\left[\bigcup_{t=0}^{n-k^{\prime}-1} R_{t}^{D}\left(\left\{v_{i}\right\}\right)\right]\right| \geq n-d+\mu
$$

It follows that there are at least $\mu$ loop vertices $u_{1}, u_{2}, \ldots, u_{\mu}$ such that $u_{i} \in$ $\bigcap_{i=1}^{\lambda}\left[\bigcup_{t=0}^{n-k^{\prime}-1} R_{t}^{D}\left(\left\{v_{i}\right\}\right)\right]$, where $i=1,2, \ldots, \mu$. That is to say,

$$
\left|\bigcap_{i=1}^{\lambda} R_{n-k^{\prime}-1}^{D}\left(\left\{v_{i}\right\}\right)\right| \geq \mu
$$

Therefore, $k(D, \lambda, \mu) \leq n-\left\lceil\frac{d-\mu+1}{\lambda}\right\rceil$.

Consider the digraph $L_{n, d}$. Let

$$
t=n-\left\lceil\frac{d-\mu+1}{\lambda}\right\rceil \text { and } k^{*}=\left\lceil\frac{d-\mu+1}{\lambda}\right\rceil \text {. }
$$

Then we consider the following two subcases.

Subcase 1. If $\lambda \leq d+1$.

When $\lambda=1$, then $t=n-d+\mu-1$. Noting that

$$
R_{t-1}^{L_{n, d}}(\{1\})=\{n-d+1, n-d+2, \ldots, n-d+\mu-1\}
$$

then

$$
\left|R_{t-1}^{L_{n, d}}(\{1\})\right|=\mu-1
$$

When $\lambda=2$, as

$$
R_{t-1}^{L_{n, d}}(\{1\})=\left\{n-d+1, \ldots, n-k^{*}\right\}
$$

and

$$
R_{t-1}^{L_{n, d}}\left(\left\{n-k^{*}-\mu+2\right\}\right)=\left\{n-k^{*}-\mu+2, \ldots, n, 1, \ldots, n-2 k^{*}-\mu+1\right\},
$$

we have

$$
\left|R_{t-1}^{L_{n, d}}(\{1\}) \cap R_{t-1}^{L_{n, d}}\left(\left\{n-k^{*}-\mu+2\right\}\right)\right|=\left|\left\{n-k^{*}-\mu+2, \ldots, n-1\right\}\right|=\mu-1 .
$$

When $\lambda=3$, if $n-2 k^{*}-\mu+1<n-d+1$, then $k^{*}=1, t=n-1$. Let

$$
X=\left\{1, n-k^{*}-\mu+2, u\right\} \subseteq V\left(L_{n, d}\right),
$$

where $u \in V\left(L_{n, d}\right) \backslash\left\{1, n-k^{*}-\mu+2\right\}$. Then $|X|=3$. Since

$$
R_{t-1}^{L_{n, d}}(\{1\})=\left\{n-d+1, \ldots, n-k^{*}\right\}
$$


and

$$
R_{t-1}^{L_{n, d}}\left(\left\{n-k^{*}-\mu+2\right\}\right)=\left\{n-k^{*}-\mu+2, \ldots, n, 1, \ldots, n-2 k^{*}-\mu+1\right\},
$$

we have

$$
R_{t-1}^{L_{n, d}}(\{1\}) \cap R_{t-1}^{L_{n, d}}\left(\left\{n-k^{*}-\mu+2\right\}\right)=\left\{n-k^{*}-\mu+2, \ldots, n-k^{*}\right\} .
$$

Thus,

$$
\left|\bigcap_{x \in X} R_{t-1}^{L_{n, d}}(\{x\})\right| \leq\left|R_{t-1}^{L_{n, d}}(\{1\}) \cap R_{t-1}^{L_{n, d}}\left(\left\{n-k^{*}-\mu+2\right\}\right)\right|=\mu-1 .
$$

If $n-2 k^{*}-\mu+1 \geq n-d+1$, then $n-2 k^{*}-\mu+1<n-d+k^{*}+1<n-k^{*}-\mu+2$. Let

$$
X=\left\{1, n-k^{*}-\mu+2, n-d+k^{*}\right\} \subseteq V\left(L_{n, d}\right),
$$

where $u \in V\left(L_{n, d}\right) \backslash\left\{1, n-k^{*}-\mu+2\right\}$. Then $|X|=3$. Since

$$
\begin{aligned}
& R_{t-1}^{L_{n, d}}(\{1\})=\left\{n-d+1, \ldots, n-k^{*}\right\} \\
& R_{t-1}^{L_{n, d}}\left(\left\{n-d+k^{*}+1\right\}\right)=\left\{n-d+k^{*}+1, \ldots, n, 1, \ldots, n-d\right\}
\end{aligned}
$$

and

$$
R_{t-1}^{L_{n, d}}\left(\left\{n-k^{*}-\mu+2\right\}\right)=\left\{n-k^{*}-\mu+2, \ldots, n, 1, \ldots, n-2 k^{*}-\mu+1\right\},
$$

we have

$$
\bigcap_{x \in X} R_{t-1}^{L_{n, d}}(\{x\})=\left\{n-k^{*}-\mu+2, \ldots, n-k^{*}\right\}
$$

which implies

$$
\left|\bigcap_{x \in X} R_{t-1}^{L_{n, d}}(\{x\})\right|=\mu-1
$$

When $\lambda=4$, if $n-2 k^{*}-\mu+1<n-d+1$, then $k^{*}=1$ and $t=n-1$. Let

$$
X=\left\{1, n-\mu+1, u_{1}, u_{2}\right\} \subseteq V\left(L_{n, d}\right),
$$

where $u_{1}, u_{2} \in V\left(L_{n, d}\right) \backslash\{1, n-\mu+1\}$. Then $|X|=4$. Since

$$
R_{t-1}^{L_{n, d}}(\{1\})=\{n-d+1, \ldots, n-\mu+1, \ldots n-1\}
$$

and

$$
R_{t-1}^{L_{n, d}}(\{n-\mu+1\})=\{n-\mu+1, \ldots, n, 1, \ldots, n-\mu-1\},
$$


we have

$$
\left|R_{t-1}^{L_{n, d}}(\{1\}) \cap R_{t-1}^{L_{n, d}}(\{n-\mu+1\})\right|=|\{n-\mu+1, \ldots, n-1\}|=\mu-1 .
$$

This implies

$$
\mid \bigcap_{x \in X} R_{t-1}^{L_{n, d}}(\{x\}) \leq \mu-1 .
$$

If $n-d+k^{*}+1>n-2 k^{*}-\mu+1 \geq n-d+1$, letting

$$
X=\left\{1, n-k^{*}-\mu+2, n-d+k^{*}+1, w\right\} \subseteq V\left(L_{n, d}\right),
$$

where $w \in V\left(L_{n, d}\right) \backslash\left\{1, n-k^{*}-\mu+2, n-d+k^{*}+1\right\}$, we have $|X|=4$. Since

$$
\begin{aligned}
& R_{t-1}^{L_{n, d}}(\{1\})=\left\{n-d+1, \ldots, n-k^{*}\right\}, \\
& R_{t-1}^{L_{n, d}}\left(\left\{n-d+k^{*}+1\right\}\right)=\left\{n-d+k^{*}+1, \ldots, n, 1, \ldots, n-d\right\}, \\
& R_{t-1}^{L_{n, d}}\left(\left\{n-\mu-k^{*}+2\right\}\right)=\left\{n-\mu-k^{*}+2, \ldots, n, 1, \ldots, n-2 k^{*}-\mu+1\right\},
\end{aligned}
$$

we have

$$
R_{t-1}^{L_{n, d}}(\{1\}) \cap R_{t-1}^{L_{n, d}}\left(\left\{n-\mu-k^{*}+2\right\}\right) \cap R_{t-1}^{L_{n, d}}\left(\left\{n-d+k^{*}+1\right\}\right)=\left\{n-k^{*}-\mu+2, \ldots, n-k^{*}\right\} .
$$

Thus,

$$
\left|\bigcap_{x \in X} R_{t-1}^{L_{n, d}}(\{x\})\right| \leq\left|R_{t-1}^{L_{n, d}}(\{1\}) \cap R_{t-1}^{L_{n, d}}\left(\left\{n-\mu-k^{*}+2\right\}\right) \cap R_{t-1}^{L_{n, d}}\left(\left\{n-d+k^{*}+1\right\}\right)\right| \leq \mu-1 .
$$

If $n-d+k^{*}+1 \leq n-2 k^{*}-\mu+1$, letting

$$
X=\left\{1, n-d+k^{*}+1, n-k^{*}-\mu+2, n-2 k^{*}-\mu+2\right\} \subseteq V\left(L_{n, d}\right),
$$

we have $|X|=4$. Since

$$
\begin{aligned}
& R_{t-1}^{L_{n, d}}(\{1\})=\left\{n-d+1, \ldots, n-k^{*}\right\}, \\
& R_{t-1}^{L_{n, d}}\left(\left\{n-d+k^{*}+1\right\}\right)=\left\{n-d+k^{*}+1, \ldots, n, 1, \ldots, n-d\right\}, \\
& R_{t-1}^{L_{n, d}}\left(\left\{n-k^{*}-\mu+2\right\}\right)=\left\{n-\mu-k^{*}+2, \ldots, n, 1, \ldots, n-2 k^{*}-\mu+1\right\}, \\
& R_{t-1}^{L_{n, d}}\left(\left\{n-2 k^{*}-\mu+2\right\}\right)=\left\{n-2 k^{*}-\mu+2, \ldots, n, 1, \ldots, n-3 k^{*}-\mu+1\right\},
\end{aligned}
$$

we have

$$
\left|\bigcap_{x \in X} R_{t-1}^{L_{n, d}}(\{x\})\right|=\left|\left\{n-k^{*}-\mu+2, n-k^{*}-\mu+3, \ldots, n-k^{*}\right\}\right|=\mu-1 .
$$


When $\lambda \geq 5$, if $n-2 k^{*}-\mu+1<n-d+1$, letting

$$
X=\left\{1, n-k^{*}-\mu+2, v_{1}, \ldots, v_{\lambda-2}\right\} \subseteq V\left(L_{n, d}\right),
$$

where $v_{i} \in V\left(L_{n, d}\right) \backslash\left\{1, n-k^{*}-\mu+2\right\}$ for $i=1,2, \ldots, \lambda-2$, then $|X|=\lambda$. Since

$$
R_{t-1}^{L_{n, d}}(\{1\})=\left\{n-d+1, \ldots, n-k^{*}\right\}
$$

and

$$
R_{t-1}^{L_{n, d}}\left(\left\{n-k^{*}-\mu+2\right\}\right)=\left\{n-\mu-k^{*}+2, \ldots, n, 1, \ldots, n-2 k^{*}-\mu+1\right\},
$$

we have

$$
\left(\bigcap_{x \in X} R_{t-1}^{L_{n, d}}(\{x\}) \subseteq\left(R_{t-1}^{L_{n, d}}(\{1\}) \cap R_{t-1}^{L_{n, d}}\left(\left\{n-\mu-k^{*}+2\right\}\right)\right)=\left\{n-k^{*}-\mu+2, \ldots, n-k^{*}\right\},\right.
$$

which implies that

$$
\left|\bigcap_{x \in X} R_{t-1}^{L_{n, d}}(\{x\})\right| \leq \mu-1
$$

If $n-d+k^{*}+1>n-2 k^{*}-\mu+1 \geq n-d+1$, letting

$$
X=\left\{1, n-d+k^{*}+1, n-k^{*}-\mu+2, v_{1}, \ldots, v_{\lambda-3}\right\} \subseteq V\left(L_{n, d}\right),
$$

where $v_{i} \in V\left(L_{n, d}\right) \backslash\left\{1, n-d+k^{*}+1, n-k^{*}-\mu+2\right\}$, for $i=1,2, \ldots, \lambda-3$, then $|X|=\lambda$. As

$$
\begin{aligned}
& R_{t-1}^{L_{n, d}}(\{1\})=\left\{n-d+1, \ldots, n-k^{*}\right\}, \\
& R_{t-1}^{L_{n, d}}\left(\left\{n-d+k^{*}+1\right\}\right)=\left\{n-d+k^{*}+1, \ldots, n, 1, \ldots, n-d\right\},
\end{aligned}
$$

and

$$
R_{t-1}^{L_{n, d}}\left(\left\{n-k^{*}-\mu+2\right\}\right)=\left\{n-\mu-k^{*}+2, \ldots, n, 1, \ldots, n-2 k^{*}-\mu+1\right\},
$$

we have

$$
R_{t-1}^{L_{n, d}}(\{1\}) \cap R_{t-1}^{L_{n, d}}\left(\left\{n-\mu-k^{*}+2\right\}\right) \cap R_{t-1}^{L_{n, d}}\left(\left\{n-d+k^{*}+1\right\}\right)=\left\{n-k^{*}-\mu+2, \ldots, n-k^{*}\right\} .
$$

Thus,

$$
\left|\bigcap_{x \in X} R_{t-1}^{L_{n, d}}(\{x\})\right| \leq\left|R_{t-1}^{L_{n, d}}(\{1\}) \cap R_{t-1}^{L_{n, d}}\left(\left\{n-\mu-k^{*}+2\right\}\right) \cap R_{t-1}^{L_{n, d}}\left(\left\{n-d+k^{*}+1\right\}\right)\right|=\mu-1 .
$$

If $n-(r+1) k^{*}-\mu+1<n-d+k^{*}+1 \leq n-r k^{*}-\mu+1$ and $2 \leq r \leq \lambda-3$, letting

$$
Y=\left\{1, n-d+k^{*}+1, n-k^{*}-\mu+2, n-2 k^{*}-\mu+2, \ldots, n-r k^{*}-\mu+2\right\} \subseteq V\left(L_{n, d}\right),
$$


and

$$
X=\left(Y \cup\left\{v_{1}, \ldots, v_{\lambda-r-2}\right\}\right) \subseteq V\left(L_{n, d}\right),
$$

where $v_{i} \in V\left(L_{n, d}\right) \backslash\left\{1, n-d+k^{*}+1, n-k^{*}-\mu+2, n-2 k^{*}-\mu+2, \ldots, n-r k^{*}-\mu+2\right\}$ for $i=1,2, \ldots, \lambda-r-2$, then $|X|=\lambda$. Since

$$
\begin{aligned}
& R_{t-1}^{L_{n, d}}(\{1\})=\left\{n-d+1, \ldots, n-k^{*}\right\}, \\
& R_{t-1}^{L_{n, d}}\left(\left\{n-d+k^{*}+1\right\}\right)=\left\{n-d+k^{*}+1, \ldots, n, 1, \ldots, n-d\right\},
\end{aligned}
$$

and for $1 \leq i \leq r$,

$$
R_{t-1}^{L_{n, d}}\left(\left\{n-\mu-i k^{*}+2\right\}\right)=\left\{n-\mu-i k^{*}+2, \ldots, n, 1, \ldots, n-(i+1) k^{*}-\mu+1\right\},
$$

we have

$$
\bigcap_{x \in Y} R_{t-1}^{L_{n, d}}(\{x\})=\left\{n-k^{*}-\mu+2, \ldots, n-k^{*}\right\} .
$$

Thus,

$$
\left|\bigcap_{x \in Y} R_{t-1}^{L_{n, d}}(\{x\})\right| \leq\left|\bigcap_{x \in X} R_{t-1}^{L_{n, d}}(\{x\})\right|=\mu-1 .
$$

If $n-d+k^{*}+1 \leq n-(\lambda-2) k^{*}-\mu+1$, letting

$$
X=\left\{1, n-d+k^{*}+1, n-k^{*}-\mu+2, n-2 k^{*}-\mu+2, \ldots, n-(\lambda-2) k^{*}-\mu+2\right\} \subseteq V\left(L_{n, d}\right),
$$

we have $|X|=\lambda$. Since

$$
\begin{aligned}
& R_{t-1}^{L_{n, d}}(\{1\})=\left\{n-d+1, \ldots, n-k^{*}\right\}, \\
& R_{t-1}^{L_{n, d}}\left(\left\{n-d+k^{*}+1\right\}\right)=\left\{n-d+k^{*}+1, \ldots, n, 1, \ldots, n-d\right\},
\end{aligned}
$$

for $1 \leq i \leq \lambda-2$

$$
R_{t-1}^{L_{n, d}}\left(\left\{n-\mu-i k^{*}+2\right\}\right)=\left\{n-\mu-i k^{*}+2, \ldots, n, 1, \ldots, n-(i+1) k^{*}-\mu+1\right\},
$$

and $n-d+k^{*}+1>n-(\lambda-1) k^{*}-\mu+1$, we have

$$
\bigcap_{x \in Y} R_{t-1}^{L_{n, d}}(\{x\})=\left\{n-k^{*}-\mu+2, \ldots, n-k^{*}\right\} .
$$

Therefore,

$$
\left|\bigcap_{y \in Y} R_{t-1}^{L_{n, d}}(\{y\})\right| \leq\left|\bigcap_{x \in X} R_{t-1}^{L_{n, d}}(\{x\})\right|=\mu-1 .
$$


From the above, we have $k\left(L_{n, d}, \lambda, \mu\right) \geq n-\left\lceil\frac{d-\mu+1}{\lambda}\right\rceil$, it follows that $k\left(L_{n, d}, \lambda, \mu\right)=n-$ $\left\lceil\frac{d-\mu+1}{\lambda}\right\rceil$.

Subcase 2. If $\lambda>d+1$.

If $\lambda>d+1$, then $t=n-1$. It is easy to see that

$$
\begin{aligned}
& R_{t-1}^{L_{n, d}}(\{1\})=\{n-d+1, n-d+2, \ldots, n-1\}, \\
& R_{t-1}^{L_{n, d}}(\{2\})=\{n-d+1, n-d+2, \ldots, n\},
\end{aligned}
$$

for $3 \leq i \leq \lambda-d$,

$$
R_{t-1}^{L_{n, d}}(\{\lambda-d\})=\{n-d+1, \ldots, n, 1, \ldots, \lambda-d-2\}
$$

and for $i=n-d+1, \ldots, n$,

$$
R_{t-1}^{L_{n, d}}(\{i\})=\{i, \ldots, n, 1, \ldots, i-2\}
$$

Let

$$
X_{1}=\{1, \ldots, \lambda-d, n-d+1\}, \quad X_{2}=\{n-d+2, \ldots, n\} \quad \text { and } \quad X=X_{1} \cup X_{2} \text {. }
$$

Then $|X|=\lambda$. Since

$$
\bigcap_{x \in X_{1}} R_{t-1}^{L_{n, d}}(\{x\})=\{n-d+1, n-d+2, \ldots, n-1\}
$$

and

$$
\bigcap_{x \in X_{2}} R_{t-1}^{L_{n, d}}(\{x\})=\{n, 1, \ldots, n-d-1\},
$$

we have $\bigcap_{x \in X} R_{t-1}^{L_{n, d}}(\{x\})=\phi$. Therefore,

$$
k\left(L_{n, d}, \lambda, \mu\right) \geq n-\left\lceil\frac{d-\mu+1}{\lambda}\right\rceil,
$$

it follows that

$$
k\left(L_{n, d}, \lambda, \mu\right)=n-\left\lceil\frac{d-\mu+1}{\lambda}\right\rceil .
$$

Case 2. If $\mu>d+1$.

Let $X \subseteq V(D)$ be a vertex set of any $\lambda$ vertices. Set $X=\left\{v_{1}, v_{2}, \ldots, v_{\lambda}\right\}$. For any vertex $v_{i} \in X$, since $D \in P_{n}(d) \subseteq P_{n}$, by Lemma 3.2,

$$
\left|\bigcup_{t=0}^{n-1} R_{t}^{D}\left(\left\{v_{i}\right\}\right)\right| \geq n-1+1=n,
$$


where $i=1,2, \ldots, \lambda$. Therefore, each loop vertex $u_{i} \in \bigcup_{t=0}^{n-1} R_{t}^{D}\left(\left\{v_{i}\right\}\right)$, where $i=1,2, \ldots, d$. Then

$$
\left\{u_{1}, u_{2}, \ldots, u_{d}\right\} \subseteq\left(\bigcap_{i=1}^{\lambda} R_{n-1}^{D}\left(\left\{v_{i}\right\}\right)\right) .
$$

Since $u_{1}, u_{2}, \ldots, u_{d}$ are loop vertices, there are at least $\mu-d$ vertices $w_{1}, w_{2}, \ldots, w_{\mu-d}$ and $w_{i} \notin\left\{u_{1}, u_{2}, \ldots, u_{d}\right\}$ such that

$$
\left\{w_{1}, w_{2}, \ldots, w_{\mu-d}\right\} \subseteq R_{\mu-d}^{D}\left(\left\{u_{1}, u_{2}, \ldots, u_{d}\right\}\right),
$$

where $i=1,2, \ldots, \mu-d$. It follows that

$$
\left|\bigcap_{i=1}^{\lambda} R_{n+\mu-d+1}^{D}\left(\left\{v_{i}\right\}\right)\right| \geq d+\mu-d=\mu .
$$

We thus arrive at

$$
k(D, \lambda, \mu) \leq n+\mu-d-1 .
$$

Next we consider the digraph $L_{n, d}$. Let $X \subseteq L_{n, d}$ be a vertex set of $\lambda$ vertices and set $X=\{1,2, \ldots, \lambda\}$. Let $t=n+\mu-d-1$. Since for $i=2, \ldots, n-d$,

$$
R_{t-1}^{L_{n, d}}(\{1\})=\{n-d+1, \ldots, n, 1, \ldots, \mu-d-1\} \subseteq R_{t-1}^{L_{n, d}}(\{i\}),
$$

and for $j=n-d+1, \ldots, n$,

$$
R_{t-1}^{L_{n, d}}(\{j\})=\{1, \ldots, n\},
$$

we have

$$
\bigcap_{x \in X} R_{t-1}^{L_{n, d}}(\{x\})=\{n-d+1, \ldots, n, 1, \ldots, \mu-d-1\},
$$

which implies that

$$
\left|\bigcap_{x \in X} R_{t-1}^{L_{n, d}}(\{x\})\right|=\mu-1 .
$$

Therefore, $k\left(L_{n, d}, \lambda, \mu\right) \geq n+\mu-d-1$. It follows that

$$
k\left(L_{n, d}, \lambda, \mu\right)=n+\mu-d-1 .
$$

Combining the proofs of Cases 1 and 2, the theorem follows as expected.

Theorem 3.4 Let $D \in P_{n}$ with girth s. Then

$$
k(D, \lambda, \mu) \leq \begin{cases}n-s+\left(n-1-\left\lfloor\frac{n-\mu}{\lambda}\right\rfloor\right) s, & \lambda \leq s, \\ n-s+\left(n-1-\left\lfloor\frac{n-\mu}{s}\right\rfloor\right) s, & \lambda>s .\end{cases}
$$


Proof Let $C_{s}$ be a directed cycle of length $s$ in $D^{(s)}$. Consider the digraph $D^{(s)}$. Choose any $r$ vertices $w_{1}, w_{2}, \ldots, w_{r}$ of $C_{s}$. Let $\frac{n-\mu}{r}=k+\frac{b}{r}$ where $0 \leq b<r-1$. In $D^{(s)}$, since $w_{i}$ is a loop vertex, $\left|R_{n-k-1}^{D^{s}}\left\{w_{i}\right\}\right| \geq n-k=n-\frac{n-\mu}{r}+\frac{b}{r}$ where $i=1,2, \ldots, r$. Therefore

$$
\left|\bigcap_{i=1}^{r} R_{n-k-1}^{D^{s}}\left\{w_{i}\right\}\right| \geq r\left(n-\frac{n-\mu}{r}+\frac{b}{r}\right)-(r-1) n=\mu+b \geq \mu .
$$

It follows that

$$
\left|\bigcap_{i=1}^{r} R_{(n-k-1) s}^{D}\left\{w_{i}\right\}\right| \geq \mu .
$$

For any $\lambda$ vertices $v_{1}, v_{2}, \ldots, v_{\lambda} \in V(D)$, there is a walk of length $n-s$ from $v_{i}$ to a vertex $u_{i}$ of $C_{s}$ where $i=1,2, \ldots, \lambda$. If $\lambda \leq s$, then $\left|u_{1}, u_{2}, \ldots, u_{\lambda}\right| \leq \lambda$ and if $\lambda>s$, then $\left|u_{1}, u_{2}, \ldots, u_{\lambda}\right| \leq s$. Hence,

$$
k(D, \lambda, \mu) \leq \begin{cases}n-s+\left(n-1-\left\lfloor\frac{n-\mu}{\lambda}\right\rfloor\right) s, & \lambda \leq s \\ n-s+\left(n-1-\left\lfloor\frac{n-\mu}{s}\right\rfloor\right) s, & \lambda>s\end{cases}
$$

\section{Conclusions}

In this paper, we studied $\mu$-scrambling indices of primitive digraphs and gave some bounds for the $\lambda$ th lower and upper $\mu$-scrambling indices of primitive digraphs with $d$ loops. However, the digraphs attaining the sharp upper bounds are not determined completely. For a general given primitive digraph, its $\mu$-scrambling indices are not given. It would be nice to settle these problems in further research.

\section{Acknowledgements}

The first author wishes to thank Professor Jun He for his guidance.

\section{Funding}

The work was supported by the Scientific and Technological Research Program of Chongqing Municipal Education Commission (Grant No. KJ1600512), National Natural Science Foundation of China (Grant No. 11701058) and Team Building Project for Graduate Tutors in Chongqing (Grant No. JDDSTD201802).

Availability of data and materials

Not applicable.

\section{Competing interests}

The authors declare that they have no competing interests.

Authors' contributions

All authors contributed equally to the writing of this paper. All authors read and approved the final manuscript.

\section{Author details}

${ }^{1}$ School of Mathematics and Statistics, Chongqing Jiaotong University, Chongqing, China. ${ }^{2}$ College of Applied Mathematics, Chengdu University of Information Technology, Sichuan, China. ${ }^{3}$ School of Computing and Mathematics, Charles Sturt University, New South Wales, Australia. ${ }^{4}$ Department of Mathematics and Statistics, Georgia State University, Georgia, USA.

\section{Publisher's Note}

Springer Nature remains neutral with regard to jurisdictional claims in published maps and institutional affiliations. 
References

1. Akelbek, M., Fital, S., Shen, J.: A bound on the scrambling index of a primitive matrix using Boolean rank. Linear Algebra Appl. 431, 1923-1931 (2009)

2. Akelbek, M., Kirkland, S.: Coefficients of ergodicity and the scrambling index. Linear Algebra Appl. 430, 1111-1130 (2009)

3. Akelbek, M., Kirkland, S.: Primitive digraphs with the largest scrambling index. Linear Algebra Appl. 430, 1099-1110 (2009)

4. Brualdi, R.A., Ryser, H.J.: Combinatorial Matrix Theory. Cambridge University Press, Cambridge (1991)

5. Chen, S., Liu, B.: The scrambling index of symmetric primitive matrices. Linear Algebra Appl. 433, 1110-1126 (2010)

6. Cho, H.H., Kim, S.-R., Nam, Y.: The m-step competition graph of a digraph. Discrete Appl. Math. 105, 115-127 (2000)

7. Huang, Y., Liu, B.: Generalized scrambling indices of a primitive digraph. Linear Algebra Appl. 433, 1798-1808 (2010)

8. Kim, H.K.: Competition indices of tournaments. Bull. Korean Math. Soc. 45, 385-396 (2008)

9. Kim, H.K.: Generalized competition index of a primitive digraph. Linear Algebra Appl. 433, 72-79 (2010)

10. Liu, B.: On fully indecomposable exponent for primitive Boolean matrices with symmetric ones. Linear Multilinear Algebra 31, 131-138 (1992)

11. Zhang, L., Huang, T.-Z.: Bounds on the generalized $\mu$-scrambling indices of primitive digraphs. Int. J. Comput. Math. 89(1), 17-29 (2012)

\section{Submit your manuscript to a SpringerOpen ${ }^{\circ}$} journal and benefit from:

- Convenient online submission

Rigorous peer review

Open access: articles freely available online

High visibility within the field

- Retaining the copyright to your article 